\title{
STS: A Structural Theory of Sets
}

\author{
Alexandru BaltaG
}

\begin{abstract}
We explore a non-classical, universal set theory, based on a purely "structural" conception of sets. A set is a transfinite process of unfolding of an arbitrary (possibly large) binary structure, with identity of sets given by the observational equivalence between such processes. We formalize these notions using infinitary modal logic, which provides partial descriptions for set structures up to observational equivalence. We describe the comprehension and topological properties of the resulting set-theory, and we use it to give non-classical solutions to classical paradoxes, to prove fixed-point theorems that relate recursion and corecursion, to formalize "super-large", reflexive categories and "super-large" circular models, and to provide "natural" solutions for domain equations.
\end{abstract}

\section{Introduction}

In this paper we consider infinitary modal logic as a foundational language for set theory. The paper summarizes, from a modal logic pointof-view, most of the results contained in the author's Ph.D. Dissertation (Baltag 1998). Due to length restrictions, we eliminated almost all proofs and many related results from the present version.

Modal logic, endowed with the Barwise-Moss- semantics (defined in Barwise and Moss (1996)), is a natural tool for providing partial descriptions for set-structures. Barwise and Moss have proved that infinitary modal logic can be used to characterize every set in classical set theory $Z F C$ and in Aczel's Antifounded set theory $Z F A$. Here we apply modal logic to define a notion of observational equivalence between structures, and use this notion to axiomatize and model a non-classical universe of sets, containg a "universal set" $U$ and other "large" sets. This universe

Advances in Modal Logic, Volume 2.

M. Zakharyaschev, K. Segerberg, M. de Rijke, and H. Wansing, eds.

Copyright (C) 2001, CSLI Publications. 


\section{2 / Alexandru Baltag}

can be seen as the largest extension of the well-founded universe that still preserves the above-mentioned property of modal characterizability.

The main axiom of our system STS ("Structural Theory of Sets") is a strengthening of Peter Aczel's Antifoundation Axiom ( $A F A)$, stated in terms of modal descriptions. Our axiom of "Super-Antifoundation" $(S A F A)$ basically says that every maximally consistent class of infinitary modal sentences characterizes some set. The existential half of the axiom can be understood as a strong Reflection Principle for modal logic. It implies both very strong Comprehension principles (namely the socalled Generalized Positive Comprehension Principle proposed by Forti and Hinnion (1989), and Forti and Honsell (1992a), and Strong Infinity axioms (e.g. the existence of inaccessible cardinals, Mahlo cardinals etc.). The sets in this universe can be identified with complete theories in infinitary modal logic. Most of the definitions and proofs of relevant settheoretical properties have a modal character. The main set-theoretical operations and relations have natural modal counterparts. Our consistency proof for this theory of sets uses an infinitary generalization of the standard "canonical model" construction for modal logic. Under certain assumptions (the existence of a weakly compact cardinal), one can show that this is indeed a model for our theory. Moreover, the model is isomorphic to one of the most interesting models for a universal set theory that has been proposed in the last years, by researchers working on topological hyperuniverses (see Forti and Hinnion 1989, Forti and Honsell 1992a).

One can show the resulting universe is very well-behaved from a settheoretical point of view: it properly extends $Z F C$ and $Z F A$, in the sense of containing standard models for these theories; it is closed under classical set-theoretical operations and it actually has much stronger closure properties; it has interesting fixed point properties, that generalize and unify the known recursion and corecursion theorems for Antifounded set theory $Z F A$; as mentioned above, it satisfies very strong Comprehension principles.

This Structural Theory of Sets can be used for foundational purposes, to provide a principled explanation for the classical set-theoretical paradoxes and a justification for the axioms of $Z F C$, as well as for some "large cardinal" axioms. It provides in a uniform manner models for many recursive and corecursive domains, which could be used for giving denotational semantics for programming languages, in the Scott-de Bakker style. Our set theory can be also taken as a basis to develop category theory notions of a high level of generality and reflexivity (e.g. the category of all categories). In the same time, this universe of sets seems to be a good candidate for a general framework to study seman- 
tical paradoxes, one that would generalize the "circular model-theory" approach to paradoxes in Barwise and Etchemendy (1987), and Barwise (1996).

\section{Antifoundation and Modal Logic}

The "structure-forgetting metaphor" was proposed by Barwise and Moss (1991), as a motivating intuition for Aczel's Antifoundation Axiom (AFA) and as an alternative to the so-called "box" metaphor underlying the familiar iterative conception of set. In the iterative picture, sets were built in stages (corresponding to the ordinals), by putting together previously constructed sets as in a box. The structural metaphor is in some sense the "dual" of the box metaphor: sets are seen to be the result of a process of successive decompositions of a given object, successive unfoldings of its internal structure. The idea is that sets are what is left when we take an aggregate (a complex object) and we abstract everything but its structure. By forgetting the nature of the components, the only thing that remains is the aggregation/disaggregation relation between the whole and the components, i.e. the membership structure. This structure is pointed, in that it has a root: the underlying process of unfolding the structure, by successive decompositions, has a starting point, namely the very object under consideration. So we look at sets as pointed binary structures. This is the same notion as the one of a Kripke structure with a distinguished world, or a model-world pair , as it is usually called by authors working on modal logic.

One can think of this conception as turning the familiar iterative picture on its head: instead of starting at the bottom and building sets in stages, as collections of previously given objects, we are now presented, from the start, with a unified totality (an "object"), which we analyze into its constituents, which in their turn are to be analyzed... and so on. By forgetting everything but this "pattern of unfolding" we obtain a set.

Observe that under this conception there is no reason to limit the possible structures to wellfounded ones. A "possible world" containing infinitely divisible objects is surely conceivable - and this is enough for mathematics, regardless of whether the real world is "atomic" or not. So the classical Axiom of Foundation has to go. But there still are some limitations on the kind of binary structures sets are. The most important restriction comes from the very principle of abstraction we assumed: the identity of sets should be given by nothing else but the structure itself. This does not reduce set-identity to simple structural isomorphism, because of the untyped character of the set concept: the components are 


\section{4 / Alexandru Baltag}

themselves regarded as being sets. In other words, sets are structures composed of sets. In this way one obtains a notion of structural equivalence. We shall refer to this relation as observational equivalence, since we think of the above analysis as a series of observations performed on the object in question. As we shall see, there are various ways to define this notion.

Working in $Z F C^{-}$, i.e. the system obtained by eliminating the axiom of Foundation from Zermelo-Fraenkel's system $Z F C$, Peter Aczel has defined the notion of a bisimulation between two structures. Bisimilarity can be defined more generally for pointed binary structures. In the universe of $Z F^{-}$, the only binary structures available are the binary graphs, which are defined as sets of ordered pairs of objects. A pointed (binary) graph $G=\left(g_{0}, R\right)$ is a pair consisting of a binary graph $R$ and a root (or a "point") $g_{0}$. A bisimulation between two graphs is a relation $\sim$ between their nodes, having the property that if two nodes are related by $\sim$ then every immediate successor of one of the nodes is related by $\sim$ with some immediate successor of the other node. A bisimulation between two pointed graphs is just a bisimulation $\sim$ between the two graphs, which relates the two roots. Two pointed graphs are bisimilar if they are related by a bisimulation.

Bisimilarity is Aczel's notion of observational equivalence. Observe that one can associate to each set $a$ some unique pointed graph $G_{a}$, with the accessibility relation given by converse membership $\ni$, and the root given by the set $a$ itself. Then we can define two sets to be bisimilar if their corresponding graphs are bisimilar. We write $a \sim b$ to denote the fact that the sets $a$ and $b$ are bisimilar.

But there exists an alternative definition for observational equivalence, in terms of infinitary modal logic: indeed, the modal language is the natural language to describe graphs (Kripke structures) up to bisimilarity. Working inside $Z F C^{-}$, Barwise and Moss (1996) have considered the infinitary modal sentences, as partial descriptions for sets and graphs. The class $L_{\infty}$ of infinitary modal sentences is defined as the least class closed under negation $\neg$, the modal operator $\diamond$ and infinitary conjunction $\bigwedge$. The definition for truth (or satisfaction) is the familiar one for Kripke structures. Given a pointed graph $G=(g, R)$ we write $(g, R) \vDash \varphi$ instead of the standard notation $g \models_{G} \varphi$. The modal theory of a pointed graph $G$ is the class $t h(G)$ of all the modal sentences satisfied by $G$.

By transferring this definition to sets, via the above-mentioned "identification" of sets $a$ with their converse-membership graphs $G_{a}$, one obtains the: 
Barwise-Moss semantics for modal logic. The relation $a \vDash \varphi$ of satisfaction of a modal sentence $\varphi$ on a set $a$ is defined recursively by: $a \models \neg \varphi$ iff $a \not \models \varphi ; a \vDash \wedge \Phi$ iff $a \vDash \varphi$ for all $\varphi \in \Phi ; a \models \diamond \varphi$ iff $a^{\prime} \vDash \varphi$ for some $a^{\prime} \in a$. The modal, theory of a set $a$ is the class th $(a)$ of all modal sentences satisfied by $a$.

One can now define a notion of modal equivalence, or observational equivalence between structures (graphs or sets): two objects (pointed graphs or sets) are said to be observationally equivalent if they satisfy the same modal sentences; i.e. if their modal theories coincide. In this case, we write $a \equiv b$ for sets, $G \equiv G^{\prime}$ for pointed graphs, and $a \equiv G$ for observationally equivalent pairs of sets and pointed graphs. From now on we shall simply refer to the relation $\equiv$ as observational equivalence, and we shall distinguish it from Aczel's relation of bisimilarity $\sim$. In the more general context we shall consider later, the two notions are indeed distinct. On the other hand, it is well-known that in the context of $Z F C^{-}$, the modal notion of observational equivalence is equivalent to Aczel's notion of bisimilarity: In $Z F C^{-}$, two objects (graphs or sets) are observationally equivalent (in the modal sense) iff they are bisimilar.

The above considerations on set identity as observational equivalence lead naturally to the following strengthening of the classical axiom of Extensionality:

Strong Extensionality (Aczel): Observationally equivalent (bisimilar) sets are identical. In modal terms: sets are characterized by their modal theories; i.e. if $\operatorname{th}(a)=t h(b)$ then $a=b$.

This axiom basically says that sets are uniquely determined by the modal descriptions of their membership structure. This gives a clear-cut identity to Aczel's sets. The above discussion on the structural metaphor suggests that strong extensionality should be the only limitation imposed on the set structures. This leads to a maximality principle, stating that, up to bisimilarity, every pointed binary structure can be seen as a set. This is the existential half of Aczel's axiom of Antifoundation:

Existential AFA: Every pointed graph is observationally equivalent (bisimilar) to some set.

By Strong Extensionality, the set mentioned in this statement is unique. So, putting together the last two axioms, one obtains a version of Aczel's main postulate:

Antifoundation Axiom (AFA): Every pointed graph is observationally equivalent (bisimilar) to a unique set.

This statement seems to perfectly capture the structural conception of 
set: sets are just pointed graphs modulo observational equivalence (bisimilarity). Aczel's system $Z F A$ is obtained by adding the Antifoundation axiom $A F A$ to $Z F C^{-}$.

Against $Z F A$, one can argue that the acceptance of the axioms of $Z F C^{--}$, as the background of our set theory, imposes an artificial limitationof-size on the kind of structures sets are. Our understanding of binary structures as gruphs, that is sets living in the universe of $Z F C^{-}$, restricts our choice for set-structures. In particular, the axioms of Replacement and Separation force our structures to be small; the sets obtained by collapsing these structures in accordance to $A F A$ will be hereditarily small. As it was observed by Barwise and Moss in Vicious Circles, it seems that $Z F A$ can be best understood as a general theory of hereditarily small sets. Indeed, if we adopt the structural conception, then the iterative justification for the axioms of $Z F C^{-}$(in particular for Separation) is no longer available: sets are not built up from below in a wellfounded manner, but are obtained by collapsing arbitrary structures. The only justification for $Z \mathrm{FC}^{-}$comes now from the older "limitation-of-size conception of sets" (Fraenkel, von Neumann): a class is considered to be a set iff it is not too big. Indeed, to accept the axioms of Replacement and Separation means to postulate that all sets are small.

But one can argue that, from the point of view of the structural conception, the "smallness" condition looks unnatural, a simple artifact of the way we usually represent (pointed binary) structures as sets in $Z F C^{-}$. It actually goes against the "maximality" principles embodied by $A F A$, by imposing again an ad-hoc restriction to the possible setstructures. Even worse, this limitation is of a non-structural nature: size is not a structural characteristic in the sense described above, since it is not preserved by bisimilarity (or by any reasonable notion of observational equivalence). Indeed, one can construct graphs of arbitrarily large size, which are nevertheless bisimilar to a given graph with only one node. Limitation of size is hence contrary to the spirit of the "structureforgetting" metaphor.

\section{A Structural Theory of Sets: STS}

In this paper we explore what happens if we take the structural notion of set at face value, and we consequently drop any size restriction. The problem of finding such a theory was explicitly asked by J. Barwise and L. Moss in their book Vicious Circles (1996), in the chapter suggestively entitled Wanted: A Strongly Extensional Theory of Classes. As an attempt to answer this call, we present an axiomatic system STS (Structural Theory of Sets). We use the standard language of Set Theory, with 
variables for sets. Sets are not assumed to be necessarily small. We also use classes as a "manner of speaking" extensionally about unary predicates (which might not define any sets). The class of all sets is denoted by $U$. We also postulate the existence of two classes $V$ and Sat. The first is intended to be the class of all wellfounded sets and so a model of $Z F C$; the second is the relation of satisfaction of a modal formula by a set. We shall assume that the class of all sets $U$ is an extensional universe satisfying some very mild closure conditions (under singletons and finite unions). The class of wellfounded sets $V$ is needed for constructing partial descriptions for structures, i.e. to define the class $L_{\infty}$ of infinitary modal sentences. We assume $V$ satisfies all the axioms of $Z F C$. A set is said to be small if it is of the same size as some set in $V$. Inside $V$, one can define the collection $O n$ of all (von Neumann) ordinals in the usual manner.

Let us list here all the axioms mentioned above, which constitute what might be called the "classical core" of our system STS:

\section{Classical Axioms}

(A1) Extensionality

(A2) Closure of the universe $U$ under singletons and finite unions: if $a, b$ are sets then $\{a\}, a \cup b$ are sets.

(A3) A set is in $V$ iff all its elements are in $V$. In other words, the class $V$ is transitive and closed under subsets: $\mathcal{P} V=V$.

(A4) $V$ is a model of the axioms of $Z F C$.

Let us mention here that for the resulting system, obtained by adding the axioms mentioned in the next section, we do not need all the assumptions included in the axiom (A4) above. Namely, only need to assume that $V$ satisfies the classical axioms of Infinity, Replacement, Union and Choice. The fact that $V$ will also satisfy the other axioms of $Z F C$ follows from our main axiom $S A F A$ (to be introduced in the next section).

Next, we define our notion of (binary) structure. By dropping the limitation-of-size condition, we return to the pre-set-theoretical ("logical") notion of structure: a binary structure is just a binary relation $R$, given by some formula. Set-theoretically, this can be still represented as a class of pairs, but not necessarily as a set, be it small or not. This is a completely naive conception of structure. If we also fix a "root", we obtain the class-analogue of a pointed graph: the notion of pointed system. This concept has been studied in the context of $Z F C$ and $Z F A$, but not as a central notion. We shall take it as the basic logical notion underlying the concept of set.

Formally, we define a pointed system as( a pair of a "root" set and) 


\section{8 / Alexandru Baltag}

a class of pairs (i.e. a binary relation, not necessarily representable by a set). A (pointed) graph is a small (pointed) system. One can easily see that every (pointed) $V$-graph is isomorphic to a (pointed) graph that belongs to $V$.

Let us now suppose for a moment that we agreed on a suitable definition of the notion of observational equivalence between pointed systems. In the next section, we will discuss this notion and argue that, in the case of possibly large pointed systems, the useful concept of equivalence is given by modal equivalence, and not by the usual definition of bisimulation. But we postpone this discussion for now and just assume as given a notion of observational equivalence.

The essence of the "unrestricted" structural conception of set can be now captured by the following statement:

Sets are just arbitrary pointed systems modulo observational equivalence.

In our system $S T S$, this will be expressed by the following $A F A$-like theorem:

Weak SAFA: Every pointed system is observationally equivalent to a unique set.

This was our initial formulation of our main axiom $S A F A$ ("Super-Antifoundation Axiom"), designed to replace $A F A$ in the context of a theory of arbitrarily-sized strongly extensional classes. We shall call this statement Weak $S A F A$, since it is weaker than the final formulation of $S A F A$ (to be presented below). The uniqueness half of Weak $S A F A$ is just (the modal version of) Strong Extensionality: observationally equivalent sets are identical. The existential half ("every pointed system is equivalent to some set") is a strengthening of the existential $A F A$. We note here, without proof, that the correspondent formulation in terms of bisimulation is inconsistent (when stated for pointed systems in full generality), since it leads to the classical set-theoretical paradoxes! But, as we shall see, our formulation in terms of observational (modal) equivalence is consistent.

\section{Modal Descriptions and Super-Antifoundation}

Let us turn to the problem of choosing the right notion of observational equivalence between pointed systems. As announced, we claim that Aczel's bisimilarity relation captures this notion only for small systems (graphs). It is not appropriate for large systems, since it is based on the unwarranted assumption that we can use one of the systems as a 
whole to describe or simulate the other. Let us suppose we first observe the roots $g_{0}, g_{0}^{\prime}$ of the two systems; next, we are presented with an immediate successor of $g_{0}^{\prime}$ in the second system, and we have to match it with some immediate successor of $g_{0}$ in the first system. But this assumes one is given a complete picture or list of the collection of all the immediate successors of $g_{0}$. This is not a natural assumption when defining a notion of observational equivalence between large systems.

A more realistic assumption is that at each step we only have access to a list of partial descriptions of all the immediate successors. The list can only use descriptions that have already been constructed; hence, even if the collection of all the successors is large, the collection of their descriptions will be small. But this means we are not matching a node with another node, but a description of a node with another description. Both might actually refer to many distinct nodes.

After $\omega$ many steps, we might have to continue this process of unfolding the structures: we now have available more descriptions of the immediate successors of the initial roots. We obtain a transfinite sequence of unfoldings, which can be interpreted as a series of analytical experiments performed on the initial object (set or pointed system). Two objects will be observationally equivalent if they have the same pattern of unfolding.

Observe the underlying temporal metaphor: as in the iterative picture, we need a "logical" concept of Time, given by an unending succession of ordinal stages; but these are no longer stages of construction, but stages of discovery. The idealized mathematician is no longer the builder of the mathematical universe, only its explorer: sets are the "ideal record" of his activity of analyzing objects into pieces. This can be related to what Keith Devlin, in his book on The Joy of Sets (1993) wrote about Antifoundation as being based on an analytic approach to set theory, in contrast with the synthetic approach of the iterative conception.

On the other hand, one can see that the analytic approach presupposes the synthesis: the outcome of an "observation" or experiment is a partial description of the object in question. To analyze something is to actually construct a new object, as a (partial) unfolding of the initial one, a (possibly incomplete) representation of its structure. The explorer has to record somehow the results of his explorations, accumulating all his past and present information in a database: a box. Each of the data gathered in the box is itself a partial description of the intended object: another box. In this sense, the iterative universe is the "shadow" of the analytic universe, its trace of unfolding: when we explore the "real world" of sets-as-structures, we simultaneously build the wellfounded 


\section{0 / Alexandru Baltag}

universe of boxes-inside-boxes, as a way to encode the partial information gained in the first process. The stages of discovery are also stages of construction (of "theories" or databases). The wellfounded sets play here the role of "linguistic objects", descriptions of the intended (possibly non-wellfounded) structures. But in the same time they are "real" objects in themselves, being a part of the intended universe.

So we formally encode these partial descriptions as particular kinds of wellfounded sets, called (infinitary) modal sentences. These are generated by the following three basic ways of building step-by-step partial descriptions for set-structures:

(1) Negation: given a possible description $\varphi$ and an object $a$, we construct a new description $\neg \varphi$, to capture the information that $\varphi$ does not describe $a$.

(2) Conjunction: given a set $\Phi$ of descriptions of the object $a$, we accumulate all descriptions in $\Phi$ by forming their conjunction $\bigwedge \Phi$.

(3) Unfolding: given a description $\varphi$ of some member (or members) of a set $a$, we "unfold" the set $a$ by constructing a description $\diamond \varphi$, which captures the information that $a$ has some member described by $\varphi$.

Observe that the first two operations refer to sets-as-objects and generate the language of infinitary propositional logic. The third is the most basic way of "analyzing" or unfolding a set: we just unwrap the box and pick up (the description of) some thing inside. The language $L_{\infty}$ generated by these three operations is called infinitary modal logic. One can consider this logic as a fragment of $L_{\infty \omega}$, the standard first-order language with infinitary conjunctions and disjunctions. One can easily define the infinitary modal language $L_{\infty}$, by recursion inside $V$, by encoding in some canonical manner the symbols $\neg, \bigwedge, \diamond$ by some well-founded sets, and then encoding a model sentence by a string made of the codes of all its symbols.

We can define the dual operators $\bigvee, \square$ in the usual manner, and also introduce some other useful operators:

$$
\begin{aligned}
\diamond \Phi=: & \{\diamond \varphi: \varphi \in \Phi\} \\
\square \Phi=: & \{\square \varphi: \varphi \in \Phi\} \\
\varphi \wedge \psi=: & \bigwedge\{\varphi, \psi\} \\
\varphi \vee \psi=: & \bigvee\{\varphi, \psi\}
\end{aligned}
$$

One can also define these modal descriptions for pointed systems (and graphs). The resulting description relation coincides with the Kripke semantics for infinitary modal logic. We call this relation satisfaction. 
For pointed graphs in $V$ one can define the notion of satisfaction by the usual recursive definition. As mentioned above, if we identify each set $a$ with its converse-membership binary structure $G_{a}$, then this definition corresponds to the set-semantics for modal logic, (described in section 2 and) defined in the more restricted case of $Z F A$ by J. Barwise and L. Moss in Vicious Circles. In this sense, modal sentences can be seen indeed as giving partial descriptions for sets. The only problem is that we cannot make this into a formal recursive definition for arbitrary sets or pointed systems. The reason is that we have not assumed any principle of $\epsilon$-recursion for arbitrary sets or classes, and actually there is no place for such a postulate in a non-wellfounded set theory: recall that $\in$-recursion is equivalent to the Foundation axiom.

Instead of a recursive definition, we just postulate the recursive conditions corresponding to the set-semantics, as axioms. For this, we assume the existence of a special class (i.e. binary predicate) Sat, as a class of pairs $(a, \varphi)$ of sets $a$ and modal sentences $\varphi$. We shall of course write $a \models \varphi$ instead of $(a, \varphi) \in$ Sat.

\section{Satisfaction Axioms}

(A5) $a \models \neg \varphi$ iff $a \not \models \varphi$

(A6) $a \models \bigwedge \Phi$ iff $a \models \varphi$ for all $\varphi \in \Phi$

(A7) $a \models \diamond \varphi$ iff $a^{\prime} \models \varphi$ for some $a^{\prime} \in a$.

One can then easily extend this notion of satisfaction to classes and to pointed systems, in such a way that the above recursive conditions remain valid. We also define as above the modal theory th $(a)$ of the set a.

Now we can define observational equivalence as identity of all the partial descriptions:

Definition 4.1 Two "objects" (sets, classes or pointed systems) are said to be observationally equivalent if they are modally equivalent, i.e they satisfy the same infinitary modal sentences. We write $a \equiv b$ to express the fact that the sets $a$ and $b$ are observationally equivalent, and similarly we write $A \equiv B, G \equiv G^{\prime} a \equiv B, a \equiv G$ etc., for pairs of classes, pointed systems, sets and classes etc.

A modal theory is said to be consistent if all its members are satisfied by the same structure (pointed system or graph). The theory is said to be weakly consistent if all its sub-sets are consistent. The assertion that every weakly consistent theory is consistent is equivalent to a "large cardinal" assumption (that of the existence of a weakly compact cardinal).

In the previous section, we have mentioned "Weak $S A F A$ " as our initial formulation of our main axiom $S A F A$, generalizing Peter Aczel's 


\section{2 / Alexandru Baltag}

Antifoundation axiom. For set-theoretical reasons (namely the desirability of having a universe of sets closed under some specific operations, like infinitary union), one needs to strengthen Weak $S A F A$ a little bit. Namely, it is easy to see that the existential side of Weak $S A F A$ is equivalent to the claim that every consistent modal theory is satisfied by some set. From an intuitive point of view, weakly consistent theories are just theories whose consistency could be proved if we would assume the above-mentioned large cardinal hypothesis. It is thus natural to strengthen Weak $S A F A$ to obtain our main axiom:

Super-Antifoundation Axiom (SAFA) Existence: every weakly consistent modal theory is satisfied by some set.

Uniqueness (Strong Extensionality): every set is characterized by its modal theory.

This completes our axiomatic system STS.

\section{Correspondence between Sets and Modal Theories}

It is easy to see that $S A F A$ implies the following statement. The function $t h$, mapping every set $a$ to its modal theory $t h(a)$, gives a bijective correspondence between sets and maximally weakly consistent modal theories. One can make this bijection into an isomorphism, by defining an accessibility relation between theories, as in the canonical model construction in standard modal logic. For $T, S$ maximally weakly consistent theories in $L_{\infty}$, we put:

$$
T \rightarrow S \text { iff } \forall \varphi(\varphi \in S \Rightarrow \diamond \varphi \in T) .
$$

Then one can prove the following

Proposition 5.1 For all sets $a, b$, we have:

$$
a \in b \quad \text { iff } \quad t h(b) \rightarrow t h(a) .
$$

In words: the above-mentioned bijection is an isomorphism between the $\epsilon$-structure of the universe of sets and the accessibility structure between maximally weakly consistent modal theories.

So one could say that a set is just a maximally (weakly) consistent theory in infinitary modal logic. As an immediate application, we obtain a number of results, which we will now list.

The Existence of a Universal Set. Let $T$ be the following theory:

$$
T=:\{\diamond \varphi: \varphi \text { is a consistent modal sentence }\}
$$

Then it is easy to check that $T$ is a weakly consistent theory. So, by $S A F A$, there must exist some set, call it $U$, such that $U \models T$. So $T$ must be included in the characteristic theory $t h(U)$ of $U: T \subseteq t h(U)$. 
Let now $a$ be an arbitrary set. Any sentence $\varphi \in t h(a)$ is consistent (since $a \vDash \varphi$ ), and so $\diamond \varphi \in T \subseteq t h(U)$ (by the way we have defined $T$ ); hence $\diamond \varphi \in t h(U)$ for every $\varphi \in t h(a)$, which by definition means that we have $t h(U) \rightarrow t h(a)$ (since both are maximally weakly consistent theories). By the above result, this means that $a \in U$, but $a$ is just an arbitrary set, so the set $U$ coincides with the universal class: $U=\{x: x$ is a set $\}$. Consistency of STS: The Canonical Model. The above-mentioned correspondence gives the idea for proving the consistency of our system $S T S$ : working in $Z F C$ with an appropriate large cardinal assumption (the existence of a weakly compact cardinal $\kappa$ ), we can define the modal logic $L_{\kappa}$ having only conjunctions of size less than $\kappa$, construct its canonical model formed of all maximally weakly consistent theories of size at most $\kappa$, and interpret the canonical model as a universe of sets, with membership defined by the accessibility relation. This construction gives a model of the system STS. We skip the details of the construction, which can be found in the author's Ph.D. Dissertation (Baltag 1998).

Modal Definability

Definition 5.2 For a modal theory (class of modal formulas) $\Phi$, the class defined by $\Phi$ is the class $\{x: x \models \Phi\}$. A class is said to be modally definable if it is the class defined by some theory $\Phi$.

Notations. For modal theories $\Phi$ and $\Psi$, we use the following notations

$$
\begin{aligned}
\square \Phi=: & \{\square \varphi: \varphi \in \Phi\} \\
\Phi^{-\square}=: & \{\varphi: \square \varphi \in \Phi\} \\
\Phi^{-\diamond}=: & \{\varphi: \diamond \varphi \in \Phi\} \\
\Phi \wedge \Psi=: & \{\varphi \wedge \psi: \varphi \in \Phi, \psi \in \Psi\} \\
\Phi \vee \Psi=: & \{\varphi \vee \psi: \varphi \in \Phi, \psi \in \Psi\} \\
\Phi_{\square}=: & \square\left(\Phi^{-} \square\right) \\
\Phi_{\diamond}=: & \diamond\left(\Phi^{-\diamond}\right) \\
D \Phi=: & \square \Phi \cup\{\diamond \varphi:(\neg \varphi) \notin \Phi\} \\
c l(\Phi)=: & \{\psi: \Phi \models \psi\} \text { (the deductive closure of } \Phi)
\end{aligned}
$$

The operations $\wedge$ and $\vee$ can be generalized to infinitary ones, for indexed families of theories. $D$ is the definability operator, since one can check that

$$
A \models D \Phi \quad \text { iff } \quad A \text { is defined by } \Phi \text {. }
$$

The Comprehension Principle for Modal Theories is provable in our system:

Lemma 5.3 Every infinitary modal theory $\Phi$ defines a set $\{x: x \models \Phi\}$. 


\begin{tabular}{|c|c|c|}
\hline Sets & $\begin{array}{l}\text { Maximally consistent } \\
\text { theories (characterizabil- } \\
\text { ity) }\end{array}$ & $\begin{array}{l}\text { Deductively closed theo- } \\
\text { ries (definability) }\end{array}$ \\
\hline$a$ & $T=\operatorname{cl}(D \Phi)$ & $\Phi=\operatorname{cl}\left(T^{-} \square\right)$ \\
\hline$b$ & $S$ & $\Psi$ \\
\hline$\left\{a_{i}: i \in I\right\}$ & $\left\{T_{i}: i \in I\right\}$ & $\left\{\Phi_{i}: i \in I\right\}$ \\
\hline$\frac{\left\{a_{\alpha}: \alpha<\lambda\right\}}{\text { Operations }}$ & $\frac{\left\{I_{\alpha}: \alpha<\lambda\right\}}{\text { Axioms for the }}$ & $\frac{\left\{\Phi_{\alpha}: \alpha<\lambda\right\}}{\text { A rioms for the corre- }}$ \\
\hline with sets & $\begin{array}{l}\text { sponding characteristic } \\
\text { theory }\end{array}$ & sponding defining theory \\
\hline$\widehat{\bigcap_{i \in I} a_{i}}$ & $D\left(\bigcup_{i \in I} T_{i}^{-\square}\right)$ & $\bigcup_{i \in I} \Phi_{i}$ \\
\hline$\bigcap_{\alpha<\lambda} a_{\alpha}$ & $D\left(\bigwedge_{\alpha<\lambda} \Phi_{\alpha}^{-\sigma}\right)$ & $\bigwedge_{\alpha<\lambda} \Phi_{\alpha}$ \\
\hline$\bigcup_{\alpha<\lambda} a_{\alpha}$ & $D\left(V_{\alpha<\lambda} \Phi_{\alpha}^{-} \square\right)$ & $\bigvee_{\alpha<\lambda} \Phi_{\alpha}$ \\
\hline & $D\left(T_{\square}\right)$ & $\square \Phi$ \\
\hline Ua & $\left(T^{-\bar{\square}}\right)_{\square} \cup\left(T^{-\diamond}\right)_{\diamond}$ & $\Phi^{-\square}$ \\
\hline$\{a\}$ & $\diamond T \cup \square T$ & $D \Phi$ \\
\hline Relations & Relations & Relations \\
\hline$a \in b$ & $S^{-\square} \subseteq T\left(\right.$ or $\left.T \subseteq S^{-\diamond}\right)$ & $D \Phi \supseteq \Psi$ \\
\hline$a \subseteq b$ & 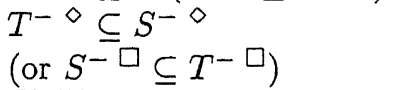 & $\Phi \supseteq \Psi$ \\
\hline
\end{tabular}

TABLE 1

But the converse is also true, showing that Modal Comprehension is enough to generate all the sets:

Lemma 5.4 Every set is definable by an (infinitary) modal theory.

Putting these together, we obtain the following characterization of setsas-classes:

Proposition 5.5 A class is a set iff it is modally definable.

This gives us a new bijection, between sets and deductively closed theories, given by mapping each set to the deductive closure of one of its defining theories: put $T h(a)=:\left\{\varphi \in L_{\infty}: b \models \varphi\right.$ for every $\left.b \in a\right\}$. This gives the announced bijection, having the inverse $T h^{-1}(\Phi)=:\{x: x \models$ $\Phi\}$ (the set defined by the theory $\Phi$ ). The theory $T h(a)$ will be called the defining theory of the set $a$, to be distinguished from the characteristic theory th $(a)$ of the set $a$ (introduced above as "the modal theory of $a$ ").

It turns out that both these two correspondences $t h$ and $T h$, "natural" set-theoretical operations correspond to "natural" operations with theories; see Table 1 .

Table 1 "shows" that the universe $U$ is closed under small unions, 
arbitrary intersections, powersets, set-unions, singletons.

Generalized Comprehension. Modal Comprehension implies apparently much stronger comprehension principles: Define the class of infinitary generalized positive formulas $\left(G P F_{\infty}\right)$ as the least class containing all atomic formulas $x \in y$, and closed under infinitary conjunctions and disjunctions, quantifiers $\forall, \exists$, and also under the following rule: if $\varphi$ is $\mathbf{G P F}_{\infty}$, and $\theta$ is any formula in $\mathbf{L}_{\infty \omega}$ which has $x$ as its only free variable, then both $\forall x \in y \varphi$ and $\forall x(\theta \rightarrow \varphi)$ are $\mathbf{G P F}_{\infty}$.

One can prove in $S T S$ the following strong comprehension theorem, which is a strengthening of a model-theoretic result by Malitz, Weydert and Forti:

GPF $_{\infty}$-Comprehension : For every formula $\varphi(x) \in \mathbf{G P F}_{\infty}$, having $x$ as a free variable, the class $\{x: \varphi(x)\}$ is a set.

As an easy consequence, the universe of sets $U$ is closed under the following operations: pairs, small unions, set-unions (i.e. if $a$ is set then $\bigcup a$ is a set), powerset, arbitrary intersections, inverse, domain, range, projections, composition, image, small Cartesian products. Another consequence is that the following relations and operations are sets (in $U$ ): identity $I=\{(x, y): x=y\}$; membership $E=\{(x, y): x \in y\}$; singleton map $S=\{(x,\{x\}): x$ is a set $\}$; the pairing function: $P=$ $\{(x, y,(x, y)): x, y$ are sets $\}$; the union function $U n=\{(x, \bigcup x): x$ is a set $\}$; the powerset function $\mathcal{P}=\{(x, \mathcal{P} x): x$ is a set $\}$; the inclusion relation $C=\{(x, y): x \subseteq y\}$; every class that includes the class of all non-hereditarily small sets( e.g.: the class of all non-wellfounded sets is a set!).

\section{Denotation of a Class}

As mentioned above, we only consider classes (and in particular pointed systems and modal theories) as ways of talking about sets. Classes are syntactic objects (predicates), which can be used to denote real objects, i.e. sets. We are trying to give a meaning, i.e. a denotation, to every definable class.

The most straightforward, naive, notion of denotation is the literal denotation. A class is thought to denote itself, in case it is a set. A class $C$ is said to have a literal denotation $d_{\text {lit }}(C)$ if and only if $C$ is a set. In this case, $C$ denotes itself: $d_{\text {lit }}(C)=C$.

Unfortunately, the literal denotation function is partial, because of the set-theoretical paradoxes: classes like the Russell class and the class of all ordinals cannot be sets, so they cannot have literal denotations. But nevertheless, $S A F A$ gives us a way to assign a unique "generalized denotation" to every class. Namely, in $S T S$, one can prove the following 


\section{6 / Alexandru Baltag}

proposition:

Every class $C$ is observationally equivalent to a unique set $d(C)$.

This is actually a particular case of Weak $S A F A$. We can explicitly define the denotation $d(C)$ by $d(C)=: t h^{-1}(t h(C))$, where $t h$ is the operator sending sets to their modal theories and $t h^{-1}$ is its inverse (which exists by the modal characterization principle, i.e. by Strong Extensionality). The operator $d$ will be called the generalized denotation (or simply the denotation) of $C$. Obviously, when $C$ is a set, the generalized denotation coincides with the literal denotation: $d(C)=d_{\text {lit }}(C)=C$.

In the context of our system, the denotation of a class is the only object (set) which is observationally equivalent to that class. So the denotation function $d$ provides us with an internal representative (inside the universe of sets) for every class. We also prove that the denotation of $C$ can be seen as the closure $\bar{C}$ of the class $C$ in a certain topology. The existence of the denotation function provides an explanation for the classical set-theoretical paradoxes. In $Z F C$ and $Z F A$, the paradoxes were understood as proving that some classes, though definable, are nevertheless meaningless, since they do not denote any object. But the proper classes in STS, while not having a literal denotation, still have a meaning, given by their generalized denotation. The lesson of the paradoxes is that we can freely define sets-as-classes only up to observational equivalence. So the Comprehension Principle is limited only by the Strong Extensionality Principle: when we "form" or define a set $d(C)$ by comprehension, denoting it by the class $C=\{x: P(x)\}$ associated to a predicate $P$, we have only identified the set up to observational equivalence. There might be many classes observationally equivalent to $C$, and the denotation function picks only one of them to represent all. Denotation is preserved by observational equivalence. But this means that $d(C)$ cannot be always equal to $C$ itself (as for the literal denotation), because distinct observationally equivalent classes must have the same denotation. The paradoxical classes are those classes $C$ for which $d(C) \neq C$.

The classical set-theoretical paradoxes can be thus understood as paradoxes of denotation. This idea was proposed, in a slightly different context, by Barwise and Moss in Vicious Circles (1996). The way we understand this proposal in $S T S$ is by the generalized denotation function. The classical paradoxes prove that the denotation of a class cannot be always "literal". This is now explained by the fact that observational equivalence puts a restriction on our power to control the actual structure of the denotation of a class. In other words: our capacity to 
define sets by predicates, or to unify classes into wholes, is subject to the limitations associated with our capacity to describe (observe) (in principle) the intended set. No definition can help us identify objects beyond the limits set by the relation of observational equivalence. The Naive Comprehension Principle is true modulo observational equivalence. The (generalized) denotation function gives us a canonical way to assign to each definable class some reference, which is an object reflecting all the infinitary modal properties of that class.

Example: An Analysis of Burali-Forti's Paradox. As an example, take Burali-Forti's Paradox: the usual way to "solve" it is by saying that the paradoxical argument shows that the class $O n$ of all ordinals is not a set. This is still true in our setting, but now the same argument gives us more information.

The Burali-Forti argument is based on the fact that the class $O n$ has all the properties of an ordinal, and so if On were a set than it would have to be an ordinal (and so it would belong to itself, contradicting its wellfoundedness). To obtain a simpler modal version of this argument, we introduce the following

Definition 6.1 Two classes $A, B$ are said to be equivalent with respect to a modal sentence $\varphi$ (or $\varphi$-equivalent) if we have: $A \models \varphi$ iff $B \models \varphi$. In this case we write $A \equiv_{\varphi} B$.

Next we show that "ordinals are more and more alike" from a modal point of view:

Lemma 6.2 Given any (infinitary) modal sentence $\varphi$, there exists some ordinal $\alpha$ such that all ordinals bigger than $\alpha$ are $\varphi$-equivalent.

Proof. Easy induction on the complexity of $\varphi$. We also remark that $\alpha$ can be taken to be the modal rank of the sentence $\alpha$. -1

As a consequence, "ordinals are more and more alike the class of all ordinals":

Lemma 6.3 Given any sentence $\varphi$, there exists some ordinal $\alpha$ such that all ordinals bigger than $\alpha$ are $\varphi$-equivalent to $O n$.

Proof. Again, this is by induction on the complexity of $\varphi$. The essential step is for the Diamond $\diamond \varphi$ and uses the previous lemma.

From this, we obtain the following

Lemma 6.4 If On were a set, then it would be observationally equivalent to $O n \cup\{O n\}$. Hence, by strong extensionality, we would have $O n=O n \cup\{O n\}$, and so $O n \in O n$, i.e. On would be itself an ordinal. 


\section{8 / Alexandru Baltag}

Proof. Observe that the hypothesis is necessary, since we can form the class $O n \cup\{O n\}$ only if we assume that $O n$ is a set.

We have to show that $O n \equiv O n \cup \dot{U}\{O n\}$, i.e. that $O n \equiv_{\varphi} O n \cup$ $\{O n\}$ for every modal sentence $\varphi$. We prove this by induction on the complexity of $\varphi$. Again, the essential step is the Diamond: suppose that $O n \models \diamond \varphi$, i.e. there exists some $\alpha \in O$ n s.t. $\alpha \models \varphi$; then we also have $\alpha \in O n \cup\{O n\}$, so we obtain that also $O n \cup\{O n\} \models \diamond \varphi$, as desired. Conversely, suppose that $O n \cup\{O n\} \models \diamond \varphi$; then, by definition of modal satisfaction, we either have that it exists some $\alpha \in O n$ s.t. $\alpha \vDash \varphi$ (in which case we immediately obtain that $O n \vDash \diamond \varphi$ and we are done) or that $O n \models \varphi$. In the second case, we know, by the previous lemma, that it exists some $\alpha \in O n$ which is $\varphi$-equivalent to $O n$. Hence, from $O n \models \varphi$, we conclude that also $\alpha \models \varphi$. But $\alpha \in O n$, so by the definition of modal satisfaction we obtain $O n \vDash \diamond \varphi$, as desired.

Corollary 6.5 On is not a set.

Proof. By definition, $O n$ is a subclass of $V$, so it is wellfounded. But, by the previous lemma, if $O n$ were a set then we would have $O n \in O n$, which contradicts wellfoundedness.

Clearly, this proof resembles Burali-Forti's argument. But it also gives more information:

Theorem 6.6 The denotation $d(O n)$ of the class $O n$ is a reflexive set:

$$
d(O n) \in d(O n)
$$

Proof. By definition, $O n \equiv d(O n)$; all the lemmas above are based on only the modal properties of $O n$, which are the same as the modal properties of $d(O n)$. Hence, we can apply all the above arguments to $d(O n)$ instead of $O n$, to conclude that: if $d(O n)$ were a set, then $d(O n)=$ $d(O n) \cup\{O n\}$ and so $d(O n) \in d(O n)$. But, by definition, $d(O n)$ must indeed be a set, so we must have $d(O n) \in d(O n)$. (We do not have a contradiction anymore, since $d(O n)$ might as well be non-wellfounded. This theorem shows that $d(O n)$ is necessarily non-wellfounded.) $\dashv$

The proof of this theorem can be further refined to give us the structure of the set $d(O n)$ :

Theorem 6.7 The denotation of the class $O n$ is a set $d(O n)$ with the following structure:

$$
d(O n)=O n \cup\{d(O n)\}
$$

So the final outcome of the Cantorian operations of successor and limit is a fixed point of these operations. The "largest ordinal" $d(O n)$ is a set that contains as members all the ordinals, and one more thing: itself. 


\section{Other Examples of Denotations of Paradoxical Classes}

- The denotation of the wellfounded universe: Let $V$ be the class of all wellfounded sets. Then $V$ is the least class which is a fixed point of the powerset operator: $\mathcal{P} V=V$. But (by Mirimanoff's Paradox) $V$ cannot be a set (since $V$ is a wellfounded class, so if it were a set it would have to be a wellfounded set, and hence we would have $V \in V$, which contradicts the wellfoundedness of $V$ ). One can show that its denotation is given by:

$$
d(V)=\{d(C): C \subseteq V\}=V \cup\{d(C): C \subseteq V \text { is a proper class }\}
$$

As a consequence, $d(V)$ is also a fixed point of the powerset operator: $d(V)=\mathcal{P} d(V)$. One can show that it is actually the least set which is a fixed point of this operator.

- The denotation of Aczel's universe: A set is said to be hereditarily small if all the sets in its transitive closure are small (or, equivalently, if its transitive closure is itself small). The class $H S$ of all hereditarily small classes is the natural model of Aczel's universe inside our theory. One can indeed prove that $H S$ is a model of $Z F A$. By the Russel's paradox, $H S$ cannot be a set, and one can easily see that its denotation is the "real" universe $U$ :

$$
d(H S)=U
$$

So Aczel's universe $H S$ of hereditarily small "hypersets" is observationally equivalent to, and yet distinct from, our reflexive universe $U$ of "supersets"! (In the terms of the above discussion, $H S$ is not really an object in itself, but just a class, i.e. a way of speaking about $U$ ).

- The denotation of Russell's class: Let $R=\{x: x \notin x\}$ be the Russell's class. By the well-known diagonal argument, $R$ cannot be a set. Its denotation turns out to be:

$$
d(R)=\{x: x \notin x \text { or } x \notin H S\}=R \cup(U \backslash H S) .
$$

\section{Topological and Closure Properties}

As mentioned above, Malitz, Weydert, Forti and Honsell , , have constructed a set-theoretical universe which is essentially isomorphic to our "canonical model" construction. Their construction is based on the intuition that sets are closed classes, where "closed" should be understood as topological closure in an appropriate topology. We recover this topology in our axiomatic system by the following:

Proposition 7.1 1. The denotation function for classes has all the properties of a topological closure operator. 


\section{0 / Alexandru Baltag}

2. A class $C$ is a set iff it is closed in this topology.

3. The topology given by denotation can be alternatively defined as being generated by the modal descriptions; i.e. a basis for this topology is given by the family of all classes of the form $\{x: x \vDash \varphi\}$, where $\varphi$ is an infinitary modal sentence.

For this reason, we alternatively use the notation $\bar{C}$ for the denotation $d(C)$ of the class $C$, and we call it the closure of $C$. The empty-set $\emptyset$ and the universe $U$ are their own closures, and so they are both sets. Closure permutes with arbitrary intersections, and as a result the universe of sets is closed under arbitrary intersections. Closure permutes with small union, and so the universe of sets is closed under small unions.

Limits. The way hypersets in $Z F A$ relate to wellfounded sets has been compared by many to the construction of the complex numbers from the reals or to the construction of the rational numbers (as pairs of integers). The way we described the sets in $S T S$, using classes of wellfounded sets (modal theories), resembles the construction of the real numbers as classes of rationals. The analogy can be pursued by studying the notion of limit that comes with the topology induced by modal descriptions. I only mention briefly some definitions and some properties.

Recall that two sets $a, b$ are said to be equivalent with respect to $a$ modal sentence $\varphi$ (write $a \equiv_{\varphi} b$ ) if we have: $a \vDash \varphi$ iff $b \models \varphi$.

Definition 7.2 Given an On-long sequence of sets indexed by ordinals $\left\langle a_{\alpha}\right\rangle_{\alpha \in O n}$ and a set $a$, we write $\lim _{\alpha \rightarrow \infty} a_{\alpha}=a$ iff for every infinitary modal sentence $\varphi$ there exists some ordinal $\delta \in O n$ such that for all $\alpha>\delta$ we have $a_{\alpha} \equiv_{\varphi} a$. In this case we say the sequence is convergent. The sequence $\left\langle a_{\alpha}\right\rangle_{\alpha \in O n}$ is said to be Cauchy iff for every modal sentence $\varphi$ there exists some ordinal $\delta \in O n$ such that for all $\alpha, \beta>\delta$ we have $a_{\alpha} \equiv{ }_{\varphi} a_{\beta}$.

As a consequence of $S A F A$, one can show that the universe $U$ is compact, as a topological space:

Compactness and Completeness. Every On-long sequence of sets $\left\{a_{\alpha}\right\}_{\alpha \in O n}$ has a convergent subsequence. As a consequence, every Cauchy sequence is convergent.

\section{Examples of Convergent Sequences}

1. the sequence of all ordinals has the denotation of "the largest ordinal" $O n$ as its limit

$$
\lim _{\alpha \rightarrow \infty} \alpha=: d(O n)=O n \cup\{d(O n)\} .
$$

2. the sequence of the classical iterative hierarchy of universes $\left(V_{\alpha}\right)_{\alpha \in O n}$, defined as usual by $V_{\alpha}=\bigcup_{\beta<\alpha} \mathcal{P}\left(V_{\beta}\right)$, converges to 
STS: A Structural Theory of Sets / 21

the (above-described) denotation of the wellfounded universe $V=$ $\bigcup_{\alpha} V_{\alpha}$ :

$$
\lim _{\alpha \rightarrow \infty} V_{\alpha}=d(V)
$$

\section{Properties of Limits}

1. The limit operator permutes with the following operations: singleton, powerset, binary union, infinitary union operator, Cartesian product.

2. The following relations are preserved under limits: $\in, \subseteq, \models, \equiv_{\varphi}$.

3. If $\lim _{\alpha \rightarrow \infty} a_{\alpha}=a$ and $\varphi$ is a modal formula then the following are equivalent:

(i) $a \models \varphi$

(ii) $\exists \alpha \forall \beta>\alpha a_{\beta} \models \varphi$

(iii) $\forall \alpha \exists \beta>\alpha a_{\beta} \vDash \varphi$

This can be generalized to $G P F_{\infty}$-formulas:

Proposition 7.3 Suppose $\lim _{\alpha \rightarrow \infty} a_{\alpha}=a$ and $\varphi(x) \in \mathbf{G P F}_{\infty}$, such that $\forall \alpha \exists \beta>\alpha \varphi\left(a_{\alpha}\right)$. Then we also have $\varphi(a)$.

Characterizations of Sets and Denotations. We can characterize the denotation $d(C)$ of a class $C$ in any of the following ways:

- By definition, $d(C)$ is the unique set which is observationally equivalent to $C$.

- $d(C)$ is the closure $\bar{C}$ of $C$ in the topology induced by modal descriptions.

- $d(C)$ is the largest class observationally equivalent to $C$.

- $d(C)$ is the least set that includes $C$. It is the "best upper approximation" of $C$ inside the universe of sets.

- An object is a member of $d(C)$ if it cannot be distinguished from all the members of $C$ by any modal formula; i.e. if it satisfies all the modal formulas true everywhere on $C$.

As a result, we have the following characterizations of the notion of set:

- A class is a set iff it is closed in the topology given by the denotation function. So the universe of sets $U$ coincides with the family of all its subclasses that are closed in this topology.

- A class is a set iff it modally definable, i.e. it is of the form $\{x$ : $x \models T$ \}, for some modal theory $T$.

- A class is a set iff it is maximal among all classes which are observationally equivalent to it. 


\section{2 / Alexandru Baltag}

Functions and Relations. We only mention a couple of results.

Proposition 7.4 Let $R \subseteq U^{n}$ be a class-relation. The following are equivalent:

1. $R$ is a set

2. $R$ is defined by a GPF $_{\infty}$-formula $\varphi\left(x_{1}, \ldots, x_{n}\right)$

3. $R$ is closed as a class with the set topology (induced by the denotation operator) on $U$

4. $R$ is closed in the product topology induced by the set topology on $U^{n}$

5. $R$ is closed under limits: if $\lim _{\alpha \rightarrow \infty} a_{\alpha i}=a_{i}$ for every $i \in\{1$, $\ldots, n\}$ and $R\left(a_{\alpha 1}, \ldots, a_{\alpha n}\right)$ holds for every $\alpha$, then $R\left(a_{1}, \ldots, a_{n}\right)$ holds.

As an application, we get:

Proposition 7.5 The universe of set-relations

$$
R e l=\bigcup_{n \in N} \mathcal{P}\left(U^{n}\right)
$$

is closed under the following operations: composition, inverse, restriction to a set, cylindrifications, small unions, arbitrary intersections; the domain and codomain of a relation are sets.

Given an equivalence set-relation $R$, any equivalence class $[x]_{R}$ is a set. For every set-relation $R$ and every object $a$, the class of all predecessors $\{x: x R a\}$ and the class of all successors $\{x: a R x\}$ of $a$ are sets.

Proposition 7.6 Let $\Gamma: U \longrightarrow U$ be a class-function. The following are equivalent:

1. $\Gamma$ is a set

2. the domain of $\Gamma$ is a set and $\Gamma$ is continuous in the set topology (defined by the denotation operator)

3. the domain of $\Gamma$ is a set and $\Gamma$ is sequential-continuous, i.e. if $\lim _{\alpha \rightarrow \infty} a_{\alpha}=a$ and all $a_{\alpha}$ 's are in the domain of $\Gamma$, then $\Gamma(a)=$ $\lim _{\alpha \rightarrow \infty} \Gamma\left(a_{\alpha}\right)$

4. the domain of $\Gamma$ is a set and $\Gamma$ is uniformly continuous in the set topology.

\section{The Problem of the Exponential}

Definition 7.7 Given two sets $a, b$, the exponential $a^{b}$ is the class of all functions having $b$ as domain and $a$ as codomain:

$$
a^{b}=:\{f: f \text { is a function }: b \longrightarrow a\} \text {. }
$$


Theorem 7.8 (Forti) $a^{b}$ is a set iff $b$ is small.

So, unlike most other set-theoretical operations, the exponential is not always defined: our universe of sets $U$ is not closed under this operation. There is no set $U^{U}$ of all total functions; moreover, there is no set $U \rightarrow U$ of all partial functions. Even worse, the exponential does not exist except in the trivial case in which the exponent is small. So we have to look for substitutes for exponentiation.

Our proposal is to restrict our attention to a particular class of functions $f: U \longrightarrow U$, that we call bounded functions. We will not formally define this notion here, since the definition is rather long and involves some complex technical notions that we have decided to skip here. It suffices to say that almost all interesting set-theoretical operators on $U$ are bounded(e.g. identity, singleton $a \longmapsto\{a\}$, powerset $a \longmapsto \mathcal{P} a$, binary union $(a, b) \longmapsto a \cup b$, set-union $a \longmapsto \bigcup a$, domain $a \longmapsto \operatorname{dom}(a)=\{x: \exists y$ s.t. $(x, y) \in a\}$, codomain, Cartesian product, permutations, projections, transitive closure, rank ) and that all the functions used outside set theory, in classical mathematics (e.g. group homorphisms) can be assumed to be bounded. Also, the composition of two bounded functions and the restriction of a bounded function to a set are bounded.

Theorem 7.9 For all sets $a, b$, the class of all total (or partial) bounded functions from $a$ to $b$ is a set.

We propose the latter as a good enough approximation of the exponential.

Definition 7.10 For sets $a, b$, define the bounded exponential to be the set of all bounded (i.e bounded by $i d+\omega$ ) functions from $b$ to $a$. We use both the set-theoretical notation $\exp _{b d}(a, b)$ and the domain-theoretic notation $b \rightarrow_{b d} a$ to denote the bounded exponential. We also consider the set of all bounded partial functions from $b$ to $a$ and we denote it by $b \rightarrow_{b d} a$.

The set $B d f=: U \rightarrow_{b d} U=\bigcup_{a, b} \exp _{b d}(a, b)$ is the set of all bounded functions. It coincides with the set of all restrictions to sets of the functions in $U \rightarrow_{b d} U$.

The (bounded) exponential operator $\exp : U \times U \longrightarrow U$ is defined by: $\exp (a, b)=: b \rightarrow_{b d} a$. The bounded application operator app is a partial function on $B d f \times U$, such that $\operatorname{app}(f, x)=: f(x)$, whenever $x \in \operatorname{dom}(f)$.

We have seen that a lot of set-theoretical functions are bounded. Now we can add more:

Proposition 7.11 The (bounded) exponential operator exp $p_{b d}$ is bounded (and hence is a function). The bounded application operator is bound- 
ed (and hence is a function). The composition operator restricted to bounded functions Comp : Bdf $\times B d f \longrightarrow B d f$, given by $\operatorname{Com}(f, g)=$ $f \circ g$, is bounded. The inverse operator restricted to boundedly injective functions is bounded. The bounded symmetric group $S_{b d}$ a of any set a is indeed a group, if considered with the composition and inverse operators.

So the notions of "bounded function" and "bounded exponential" seem to be very well-behaved approximations of their unbounded versions. The bounded exponential contains most natural set-theoretical functions. Moreover, as we shall see in the section on Applications in model theory, one can show that all the functions used outside set-theory will have bounded "copies": every first-order structure is isomorphic to a "bounded" structure (endowed only with bounded functions).

\section{Applications}

\section{Fixed Point Theorems: Recursion and Corecursion}

Definition 8.1 A monotonic class-operator is a class-function, i.e. a functional class $\Gamma$ of pairs, with the property that: $x \subseteq y \Longrightarrow \Gamma(x) \subseteq$ $\Gamma(y)$. A fixed point in $U$ (or a set-fixed point) of the operator $\Gamma$ is a set $x$ such that $\Gamma(x)=x$.

Note that every monotonic class-operator $\Gamma$ can be extended in a canonical manner to an operator on classes, by putting $\Gamma(C)=: \bigcup\{\Gamma(x)$ : $x$ is a set $\in U$ and $x \subseteq C\}$ and the resulting operator is still monotonic: $x \subseteq y \Longrightarrow \Gamma(x) \subseteq \Gamma(y)$. By extension, we can say that a class $X$ is a fixed point of $\Gamma$ if $\Gamma(X)=X$. (Of course, such a fixed point might not be in $U$, so it might not be a set-fixed point.

Proposition 8.2 If $\Gamma: U \rightarrow U$ is a monotonic operator then $\Gamma$ has a least (set-)fixed point $\Gamma_{\infty} \in U$ and a greatest (set-)fixed point $\Gamma^{\infty} \in U$.

Proposition 8.3 If $\Gamma: U \rightarrow U$ is a monotonic operator, then:

1. (The extension of) $\Gamma$ (to classes) has a least class-fixed point lf $p(\Gamma)$ and a greatest class-fixed point $g f p(\Gamma)$.

2. $\Gamma_{\infty}=\bigcap\{x: \Gamma x \subseteq x\}$

3. $\Gamma^{\infty}=g f p(\Gamma)=\bigcup\{x: x \subseteq \Gamma x\}$.

This means that the largest fixed point inside our universe of sets is the real one:

Corollary 8.4 The largest (class-)fixed point of a monotonic operator is always a set.

This is not true for least fixed points, e.g. for the powerset operator: lf $p(\mathcal{P})=V$, while $\mathcal{P}_{\infty}=d(V) \neq V$. So, in general, the greatest fixed point is better-behaved than the least fixed point. 
For monotonic functions, one can construct both the least and the greatest fixed points in $O n$ steps:

Theorem 8.5 Let $\Gamma \in U, \Gamma: U \rightarrow U$ be a monotonic set-function. Define by recursion on ordinals:

$$
\begin{aligned}
& \Gamma_{\alpha}=: \bigcup_{\beta<\alpha} \Gamma\left(\Gamma_{\beta}\right), \\
& \Gamma^{\alpha}=: \bigcap_{\beta<\alpha} \Gamma\left(\Gamma^{\beta}\right) .
\end{aligned}
$$

Then:

1. $\operatorname{lfp}(\Gamma)=\bigcup_{\alpha} \Gamma_{\alpha}$

2. $\Gamma_{\infty}=d(l f p(\Gamma))=\lim _{\alpha \rightarrow \infty} \Gamma_{\alpha}$

3. $\Gamma^{\infty}=g f p(\Gamma)=\bigcap_{\alpha} \Gamma^{\alpha}=\lim _{\alpha \rightarrow \infty} \Gamma^{\alpha}$.

Example: Take $\Gamma$ to be the powerset operator $\mathcal{P}$. This is a monotonic operator, and it is a set in our universe. The approximations $\Gamma_{\alpha}$ are the standard universes $V_{\alpha}$ of the iterative hierarchy; the least class-fixed point $l f p(\Gamma)$ is the wellfounded universe $V$, while the least set-fixed point is the denotation $d(V)$ of the wellfounded universe. Aczel's universe of all hereditarily small sets $H S$ is also a class-fixed pointof this operator. But the largest fixed point $\Gamma^{\infty}=g f p(\Gamma)$ is the universal set $U$, and in this case it is reached in just one approximation step, since $\Gamma^{0}=\Gamma^{\alpha}=U$ for all $\alpha$.

We can see that, for monotonic set-functions, the natural recursive process of approximation of the fixed points converges in at most $O n$ steps. The reason this recursive process converges to a fixed point is not that the recursion would close off at some ordinal (as in $Z F C$ ). Our set-function $\Gamma$ might be large (e.g. the powerset function $\mathcal{P}$ ), in which case no ordinal will suffice. But, as long as $\Gamma$ is a set, the recursive process will reach a fixed point in $O n$ steps. Another interesting thing is that, unlike in the case of $Z F C$ and $Z F A$, our theory relates recursion and corecursion in a simple, symmetrical manner: given a monotonic operator on sets, one can approximate the greatest fixed point by a descending sequence, dual to the one that approximates the least fixed point. This is only possible because of the presence of very large, "overcomprehensive" sets, which nevertheless remain "well-behaved" from a set-theoretical point of view.

\section{Domain Equations}

In the semantics of programming languages, one needs to solve reflexive domain equations of the following form:

$$
X=F(X) \text {. }
$$




\section{6 / Alexandru Baltag}

Usually, $F$ is assumed to be built by composing elementary operations, like union, Cartesian product, powerset, exponential etc. There are several known approaches to this problem: Scott domains, de Bakker-Rutten metric domains etc. In all of them, the "real" operations of powerset and exponentiation are replaced by "internal" operators. This is because of the size barrier imposed by Cantor's theorem: in $Z F C$ (and $Z F A$ ) we always have $|A|<|\mathcal{P} A|$ and $|A|<\left|B^{A}\right|$ (for $|B|>2$ ). This barrier has been lifted from our theory, which makes possible to find "real" solutions to reflexive equations. (This has already been observed by Forti, in the frame of his "hyperuniverses".)

Indeed, our fixed point above can be used to solve equations of the form $X=F(X)$, for functions $F$ composed of: binary (and small) unions, set-unions, powersets $\mathcal{P}$, Cartesian product, inverse $X^{-1}$, domain $\operatorname{dom}(X)$, codomain $\operatorname{cod}(X)$, relational composition $X \circ Y$, projections, image operator, bounded exponentiation with a fixed base $B \rightarrow_{b d} X$, bounded partial exponentiation $X \rightarrow_{b d} Y$. All these operators are monotonic functions in our universe, and so their compositions are also monotonic functions.

Some Examples: If $A, B$ are sets then the following equations have solutions $X$ that are sets:

$$
\begin{aligned}
& X=\mathcal{P} X \text { (both } d(V) \text { and } U \text { are solutions) } \\
& X=A \cup \mathcal{P}(B \times X) \\
& X=\mathcal{P}\left(A \times X^{-1}\right) \\
& X=A \cup\left(B \rightarrow_{b d} X\right) \\
& X=X \rightarrow_{b d} X \\
& X=A \cup\left(X \rightarrow_{b d} X\right) \\
& X=X \circ(A \cup(B \times X))
\end{aligned}
$$

\section{Model Theory}

In this subsection we explore some elementary notions of model theory inside $S T S$. We plan to develop the subject in a future paper.

Tarski's Paradox: The Universal Model. One can formalize model theory inside our system STS, in the usual manner, defining the notion of a model $\mathrm{M}$ for a given language $L$. The only problem is to define the satisfaction relation. As we shall see, there cannot be a unique formula in STS, to define truth or satisfaction of an arbitrary sentence in an arbitrary model. But one can define a formula $S a t_{n}$ that defines satisfaction for formulas of complexity length $n$. Sat $t_{n}$ is a ternary relation between models, formulas and valuation, which we shall usually write as $\mathbf{M} \models \varphi[v]$. The definition is by induction on $n$, with the obvious 
inductive clauses, starting with:

$$
\mathbf{M} \models R\left(x_{1} \ldots x_{n}\right)[v] \Longleftrightarrow:\left(v\left(x_{1}\right), \ldots, v\left(x_{n}\right)\right) \in d_{\mathbf{M}}(R),
$$

for formulas of complexity 0 , etc. We write informally $M \vDash \varphi[v]$, and that " $\varphi$ is satisfied (or true) in the model $M$ by the valuation $v$ ", whenever we have $\mathbf{M} \models_{n} \varphi[v]$ for $n=l h(\varphi)$ being the complexity length of $\varphi$. But we should keep in mind that this is not a first-order formula in STS, but a metatheoretic device to refer to a disjunction of infinitely many formulas. For particular classes of models (e.g. small models), this is actually equivalent to some first-order formula. But not in general. One can check that all the usual ways to make this inductive definition into a single formula fail in our setting, because of the failure of Separation and Replacement for "large" models.

Given a theory $T$, i.e. a set of (codes of) sentences in first-order logic, we say that " $\mathbf{M}$ is a model of the theory $T$ " if $\mathbf{M} \models \varphi$ for every $\varphi \in T$. Again, this is not a definable formula in $S T S$, but a metaformula. Nevertheless, we can still use it.

In particular, we can take now the language of set theory $L=$ $(\operatorname{Var}, \epsilon)$ and we can write the set of (codes of) axioms for STS. We shall also denote this theory by $S T S$. Notice that $S T S$ is an infinite system of axioms, since we have stated it using axiom schemes. This is similar to the case of Zermelo's $Z F C$. We can consider models $\mathbf{M}=(M, R)$ of this language, with $M$ some arbitrary set and $R \in \mathcal{P}\left(M^{2}\right)$. And then we can prove the following interesting theorem:

Theorem 8.6 (Strong Reflection Theorem for STS) The theory $S T S$ has a model. Namely the set $(U, \in)$ is a model of our theory.

This seems to contradict Tarski's theorem. In reality, it does not: we cannot use it to define truth inside the system as "satisfaction in $(U, \in)$ ", because we have not defined any single satisfaction relation. But then what is the content of the Strong Reflection Theorem?

Recall that $Z F C$ had also infinitely many axioms. In $Z F C$ there is a single definable "satisfaction relation", but no single internal model; by the Reflection Theorems, there were infinitely many "partial internal" models, for any given finite subset of the axioms. The situation in STS is completely dual: there is a single internal model for all the axioms, but no definable general notion of satisfaction; but for every $n$ we can define a formula $S a t_{n}$, which gives the satisfaction relation for all formulas of complexity less than $n$; the definition looks pretty uniform from outside the system (it is an induction), and it is clear that it agrees with the external, meta-theoretical, notion of satisfaction for all formulas of lower complexity. But there is no way to write it in a uniform way inside the 
system.

So the actual content of the above Theorem is a schema saying that: For each finite subset $T$ of the axioms of STS, let $n$ be some (externally given) natural number, larger than the complexity of all the sentences in $T$. Then we have $(U, \in) \models_{n} \wedge T$.

So we conclude that the system STS has the interesting property that it provides a definable model for itself. This model can be seen to be a model from inside the theory: the system can prove that each of its axioms holds in the universal model $(U, \in)$. What the system cannot do is to say that the universal model is a model of all its axioms; and this is because there is no uniform notion of "satisfaction (truth) of an arbitrary formula in a model".

Sets of Models of a Given Theory. We would like to have the classes of models $\operatorname{Mod}(T)$ of important first-order theories $T$ as sets in our universe. For purely relational languages, this is possible:

The class of all models of a relational language (no functions) is a set.

But it is clear that we cannot expect the functional languages to have the same property, because of the problems with the exponential operator. So we need to restrict ourselves to bounded models.

Definition 8.7 A model is bounded if the interpretations of all the function-symbols are bounded functions. We denote by $\operatorname{Mod}_{b d}(T)$ the class of all models of the theory $T$.

It is easy to see that, by restricting ourselves to bounded models, we do not lose anything from the model-theoretic point of view, as far as small models are concerned:

Proposition 8.8 Given a small model $M$, there exists a bounded model isomorphic to $M$. Moreover, there exist many such isomorphic copies: the class of all bounded models which are isomorphic to $M$ is large.

Proposition 8.9 By $\mathbf{E P F}_{\infty}$-Comprehension, the following classes of models are sets:

1. the set of all bounded models of a given signature;

2. the set of all bounded models of an equational theory;

3. the set of all bounded models of a positive theory. Here a "positive theory" is a theory whose formulas are built from atoms of the form $R t_{1} \ldots t_{n}$ and $t_{1}=t_{2}$ (equations), using conjunction, disjunction, quantifiers $\forall, \exists$ and bounded universal quantifiers of the form $\forall x_{1} \ldots x_{n}\left(R x_{1} \ldots x_{n} \rightarrow \ldots\right)$ (but no negation). 
Examples: the set of all bounded monoids, the set of all bounded groups, the set of all bounded rings, the set of all bounded vector spaces, the set of all bounded lattices (defined in terms of join and meet, not as partial orders), the set of all bounded Boolean algebras, the set of all binary (pointed) graphs (Kripke structures).

Observe that these sets of models contain copies of all the small models of the given type, but also large natural models: the model $(U, \subseteq)$ belongs to the last set above; the models $(U, U),(U, \times)$ are bounded monoids etc.

Unfortunately, the definitions of important notions like "field" and "integral domain" involve negative assertions (e.g. " $0 \neq 1$ "). But in such cases, the negativity is eliminable, by simply fixing some of the constants. If, for instance, we agree to consider only fields or integral domains having the empty set $\emptyset$ as the zero element 0 , then the classes of these algebraic structures are sets.

\section{Category Theory}

A well-known problem in the foundations of category theory is to find a way to make sense of "large categories" (e.g. the category of all sets) and of "super-large, reflexive categories" (e.g. the category of all categories). Both are forbidden by the limitation-of-size assumption built into $Z F C$ and $Z F A$. The second case (reflexive categories) is completely intractable in $Z F C$. The first case (large categories) is tractable in an indirect way, by using classes; nevertheless, this treatment makes impossible some natural categorial constructions on large categories: e.g only for small categories $A, B$, we are able to define the exponential category $[\mathbf{A}, \mathbf{B}]$ (having as objects all functors $F$ from $\mathbf{A}$ to $\mathbf{B}$, as morphisms from $F$ to $G$ all natural transformations from $F$ to $G$, as identities the identity natural transformations, and as composition the composition of natural transformations). For large categories, the exponential category is said to be illegitimate. Sometimes, these problems are solved by adding to $Z F C$ one more layer of objects ("families") on top of "classes" and "sets". A natural thing to do would be to add transfinitely many more layers, and assuming they also satisfy the axioms of $Z F C$; this is equivalent to asserting the existence of an inaccessible cardinal in $Z F C$. But of course, this only lifts the problem, without actually solving it. In particular, there will be no "reflexive" categories (i.e. categories which are among their own "objects") in any such set theory.

We present here an attempt to to deal with these problems inside our theory. The resulting category theory will be universal with respect to its objects, but it will be restricted with respect to its morphisms: only bounded functions are allowed as morphisms and functors. 


\section{0 / Alexandru Baltag}

Definition 8.10 A bounded category is a sextuple $\mathbf{A}=(\mathcal{O}$, hom, $i d, \circ$, dom, cod), consisting of:

1. a set $\mathcal{O}$, whose members are called $\mathbf{A}$-objects;

2. a bounded function hom : $\mathcal{O} \times \mathcal{O} \rightarrow U$; the members of each hom $(A, B)$ are called $\mathbf{A}$-morphisms from $A$ to $B$

3. a bounded function $i d: \mathcal{O} \rightarrow U$, such that $i d(A) \in h o m(A, A)$ for every object $A \in \mathcal{O}$; the morphism $i d(A)$ will be denoted by $i d_{A}$ and called the $\mathbf{A}$-identity on $A$;

4. a bounded binary partial function $\circ$ (composition), such that, for all A-objects $A, B, C$ and for all A-morphisms $f \in h o m(A, B), g \in$ $\operatorname{hom}(B, C)$, the function $\circ$ is defined and $g \circ f \in \operatorname{hom}(A, C)$; the morphism $g \circ f$ is called the composite of $f$ and $g$;

5. bounded partial functions dom and cod, defined on all A-morphisms into the set $\mathcal{O}$ of objects; the object $\operatorname{dom}(f)$ is called the domain of $f$, while $\operatorname{cod}(f)$ is called the codomain of $f$.

The above sets and functions are required to satisfy the following equations:

(a) composition is associative: $h \circ(g \circ f)=(h \circ g) \circ f$, whenever both are defined;

(b) A-identities act as identities with respect to composition: $i d_{B} \circ f=$ $f$ and $f \circ i d_{A}=f$, for every $f \in \operatorname{hom}(A, B)$;

(c) $\operatorname{dom}(f)=A$ and $\operatorname{cod}(f)=B$, for every $f \in h o m(A, B)$.

Observation: By $\mathbf{E P F}_{\infty}$-Comprehension, the class of all bounded categories Cat is a set.

Examples. The (bounded) category of all sets Set (having sets as objects $\mathcal{O}=U$, bounded partial functions as morphisms hom $(A, B)=$ $\exp _{b d}(B, A)=A \rightarrow_{b} d B$, functional composition as composition $\circ$, the identity function on $A$ as the identity $i d_{A}$ and the functional domain and codomain as its dom and cod functions); the category of all bounded groups Grp (having bounded groups as objects and bounded group homomorphisms as its morphisms); the category of vector spaces Vect (with vector spaces as objects and bounded linear maps as morphisms); the category of bounded topological spaces Top (with bounded topological spaces as objects and bounded continuous functions as morphisms).

Note that all these "large" categories are members of the set Cat of all bounded categories.

Definition 8.11 If $\mathrm{A}$ and $\mathrm{B}$ are bounded categories, then a bounded functor from $\mathbf{A}$ to $\mathbf{B}$ is a bounded function $F$ that assigns to each $\mathbf{A}$ object $A$ some B-object $F(A)$ and to each A-morphism $f \in \operatorname{hom}\left(A, A^{\prime}\right)$ 
some B-morphism $F(f) \in \operatorname{hom}\left(F(A), F\left(A^{\prime}\right)\right)$, in such a way that: $F$ preserves composition and identities: $F(f \circ g)=F(f) \circ F(g), F\left(i d_{A}\right)=$ $i d_{F(A)}$.

Most natural functors between bounded categories are bounded functors: e.g. the forgetful functor, the identity functor, the (covariant and contravariant) hom-functors, the (covariant and contravariant) powerset functors etc.

Definition 8.12 The category of all bounded categories Cat has as objects all the bounded categories, as morphisms from $\mathbf{A}$ to $\mathbf{B}$ all the bounded functors from $\mathbf{A}$ to $\mathbf{B}$, and as composition the usual composition of functors.

Proposition 8.13 The category Cat contains as objects all the abovementioned categories Set, Grp, Vect, Top, Cat. In particular, Cat is $a$ reflexive category: Cat $\in \mathcal{O}_{\text {Cat }}$ is an object of itself.

One can go on and define a notion of bounded natural transformation between bounded functors, and define the bounded exponential category [A, B], for any two bounded categories A, B.

\section{Games and Semantical Paradoxes}

We are currently exploring the possible treatments inside our universe of sets of the classical semantical paradoxes (e.g. the Liar), epistemic paradoxes and game-theoretic paradoxes (e.g. the so-called Hypergame paradox, see Barwise and Moss (1996) for a good presentation and a treatment inside $Z F A$ ).

Hypergame is the game with two players, in which the first move of player $A$ is to choose any well-founded game $G$ (i.e. a game in which every play ends necessarily in finitely many steps), and then they just play the game $G$ with player $B$ as the first player. The paradox is generated by the question whether hypergame is a wellfounded game or not. The solution of the paradox inside both $Z F C$ and $Z F A$ theories is that hypergame simply does not exist, because of size-restrictions: the class of all wellfounded games is not a set, and hence cannot constitute the set of all possible first-moves of player $A$. The solution in our theory is more subtle: hypergame, as defined above, still doesn't exist, but there exists a very close approximation of it, which can be understood as its denotation or closure $d$ (hypergame). The set of first-moves for player $A$ is the denotation of the class of all well-founded games (what one might call the set of quasi-wellfounded games). This game d(hypergame) is itself quasi-wellfounded, but not well-founded (since it is one of its own possible first-moves: $A$ can simply choose $G=d($ hypergame $)$ ). The relation between the ("inexistent") hypergame and its denotation, the 


\section{2 / Alexandru Baltag}

"real" game d(hypergame) is similar to the relation between the wellfounded universe $V$ and its denotation $d(V)$.

A similar solution can be given to Liar-like paradoxes. The class False of all false propositions is not a set, but just a class. Its denotation $d($ False $)$ is a set though, the set of all "almost false" propositions. The Liar sentence, as such, is not a well-defined "proposition", since it is meaningless (or paradoxical, or having only a context-dependent meaning, as in Barwise and Etchemendy (1987)). But there exists a closely related sentence $d(\operatorname{Liar})$, which is not paradoxical; this is the sentence $d($ Liar $)=:$ " $d($ Liar $)$ is almost false". This turns out to be a meaningful proposition. Moroever, this proposition $d($ Liar $)$ is true!

\section{Conclusions and Comparisons}

This paper is an attempt to build a set theory on a purely structural view on the concept of set. Our work was inspired and builds on the results and of Barwise and Moss (1996) and Aczel (1988). As we mentioned, our model for the universe of sets turned out to be isomorphic to the one obtained by and Forti and Hinnion (1989), as a model for the Generalized Positive Comprehension Principle. But their construction is just a topological construction, for which they do not provide any intuitive set-theoretical (or philosophical) justification. They have some proposals for axioms (of a topological flavor), but these axioms are neither intuitive, nor sufficient to derive all the important properties of the model. They only prove these properties (included some of the results mentioned in this paper) in the meta-theory.

In contrast, we present an axiomatic system, based on and justified by our intuitions about the notions of set, structure, modal description and observational equivalence. We propose an analytical picture, in which objects are analyzed in stages and all we can know about them are their successive partial structural descriptions, given by modal sentences. A set is what is left from this process of analysis: it is the trace of unfolding of some possible object, given by a maximally consistent class of partial descriptions. In fact, sets can be identified with the maximally consistent theories that characterize them. In this sense, a set is a canonical possible world, in the sense of the canonical model for modal logic. Sets can also be understood as modally definable classes.

We have a notion of observational equivalence between structures, defined as identity of description, i.e. modal equivalence. Sets can be understood as arbitrary (pointed binary) structures modulo observational equivalence. As collections, sets are closed, completed classes, which are as large as their modal description allows them. They contain every 
object which cannot be distinguished (separated) from all their elements by any modal property.

Our system $S T S$ of set-theoretical axioms is powerful enough to formally derive all the results about "hyperuniverses", discovered byForti and others. In our axiomatic frame, we recover their results, and we also present some new ones (including the applications on category theory and model theory). The universe of STS has nice fixed-point and closure properties. Recursion and corecursion are related in a simpler manner over this universe than over Aczel's hyperset universe. Some categorytheory notions can be stated as objects (sets), not just as classes. As already observed by Forti, this universe provides solutions to domain equations, which could be used as frameworks for denotational semantics. STS belongs to circular model theory, in the sense that it contains its own model as an object. For this reason, STS seems to be a good candidate for a general framework to study semantical paradoxes, in the spirit of the "circular-model approach" theory of Situation Theory (see Barwise 1987 and 1996).

\section{References}

Abramski, Samson. 1988. A Cook's Tour of the Finitary Non-Well-founded Sets. Unpublished manuscript.

Aczel, Peter. 1988. Non-Well-Founded Sets. CSLI Lecture Notes, No. 14. Stanford: CSLI Publications.

Baltag, Alexandru. 1995. Modal Characterizations for Sets and Kripke Structures. Unpublished manuscript.

Baltag, Alexandru. 1998. STS: A Structural Theory of Sets. Doctoral dissertation, Indiana University, Bloomington, Indiana. Available as micrifilm from Microfilms International. To be published in Kluwer's Applied Logic Series.

Barwise, Jon, and John Etchemendy. 1987. The Liar: An Essay in Truth and Circular. Oxford: Oxford University Press.

Barwise, Jon, and Lawrence Moss. 1991. Hypersets. The Mathematical Intelligencer 13(4):31-41.

Barwise, Jon, and Lawrence Moss. 1996. Vicious Circles: On the Mathematics of Non-Wellfounded Phenomena. CSLI Lecture Notes, No. 60. Stanford: CSLI Publications.

Boffa, Maurice. 1989. A Set Theory with Approximations. Jahrubuch der Kurt Gödel Gessellschaft.

Dauben, J. Warren. 1990. Georg Cantor: His Mathematics and Philosophy of the Infinite. Princeton University Press.

Devlin, Keith. 1993. The Joy of Sets. Undergraduate Texts in Mathematics. Berlin: Springer Verlag. 


\section{4 / Alexandru Baltag}

Fagin, Ronald. 1994. A Quantitative Analysis of Modal Logic. Journal of Symbolic Logic 59(1):209-252.

Forster, T. E. 1995. Set Theory with a Universal Set. Oxford Logic Guides, No. 31. Oxford: Oxford Science Publications. second edition.

Forti, Mario, and R. Hinnion. 1989. The Consistency Problem for Positive Comprehension Principles. Journal of Symbolic Logic 54(4):1401-1417.

Forti, Mario, and Furio Honsell. 1983. Set Theory with Free Construction Principles. Annali Scuola Normale Supeiore di Pisa, Classe di Scienze 10:493-522.

Forti, Mario, and Furio Honsell. 1985. Axioms of Choice and Free Construction Principles. Bull. Soc. Math. Belg. 36:69-79.

Forti, Mario, and Furio Honsell. 1992a. A General Construction of Hyperuniverses. Quadermi dell' Istituto di Matematiche Applicate "U. Dini" 9.

Forti, Mario, and Furio Honsell. 1992b. Weak foundation and antifoundation properties of positively comprehensive hyperuniverses. In L'Antifondation en Logique et en Theeorie des Ensembles, ed. R. Hinnion. 31-43. Cahiers du Centre de Logique.

Garciadiego, A. R. 1992. Bertrand Russel and the Origins of the Set-theoretical Paradoxes. Birkhauser Verlag.

Hallet, Michael. 1984. Cantorian Set Theory and Limitation of Size. Oxford: Clarendon Press.

Malitz, R. J. 1976. Set Theory in which the Axiom of Foundation Fails. Doctoral dissertation, University of California, Berkley, California. Unpublished.

Moore, A. W. 1990. The Infinite. Problems of Philosophy. Routledge.

Moschovakis, Yiannis N. 1994. Set Theory Notes. Undergraduate Texts in Mathematics. Amsterdam: North Holland.

Weydert, F. 1989. How to Approximate the Naive Comprehension Principle Inside Of Classical Logic. Bonner Math. Schriften 194. 